\title{
Efficacy of single-incision needle-knife biopsy for sampling subepithelial lesions
}

\section{(ㅇ) $\circledast \odot$}

\author{
Authors \\ Yuto Shimamura ${ }^{1}$, Jason Hwang ${ }^{1,2}$, Maria Cirocco ${ }^{1}$, Gary R. May ${ }^{1}$, \\ Jeffrey Mosko ${ }^{1}$, Christopher W. Teshima ${ }^{1}$ \\ Institutions \\ 1 Division of Gastroenterology, St. Michael's Hospital, University \\ of Toronto, Toronto, Ontario, Canada \\ 2 Division of Gastroenterology, The Wesley Hospital, \\ Auchenflower, Australia
}

submitted 27.5.2016

accepted after revision 17.11 .2016

Bibliography

DOI http://dx.doi.org/10.1055/s-0042-122334 |

Endoscopy International Open 2017; 05: E5-E10

(c) Georg Thieme Verlag KG Stuttgart · New York ISSN 2364-3722

Corresponding author

Christopher Teshima, MD, MSc, PhD, Division of Gastroenterology, St. Michael's Hospital, University of Toronto, 30 Bond Street,

Toronto, Ontario M5B1W8, Canada

TeshimaC@smh.ca

\begin{abstract}
Background and study aims Single-incision needle-knife (SINK) biopsy is a diagnostic method for acquiring tissue samples for subepithelial lesions (SELs). A single linear incision is made in the overlying mucosa and tissue samples are obtained by passing conventional biopsy forceps through the opening and deep into the lesion. The aim of this study was to describe the efficacy and safety of this technique.

Patients and methods Consecutive patients who underwent SINK biopsy for an upper gastrointestinal SEL between October 2013 and September 2015 were retrospectively reviewed.

Results Forty-nine patients underwent 50 SINK biopsies. Sufficient sampling for a definite pathologic diagnosis was obtained in $42(86 \%)$ cases, with $91 \%(40 / 44)$ having sufficient sample to perform immunohistochemistry when deemed clinically relevant. Of the 26 patients with prior non-diagnostic biopsies or FNA, a specific diagnosis was obtained in $85 \%$ (22/26). There were no significant adverse events.

Conclusions SINK biopsy is a safe and feasible strategy for obtaining a definitive tissue diagnosis with immunohistochemistry for SELs.
\end{abstract}

\section{Introduction}

Obtaining a definitive diagnosis for subepithelial lesions (SELs) is often challenging because biopsies of the overlying surface mucosal layer are typically normal. Jumbo forceps biopsy, biteon-bite tunneling technique with conventional biopsy forceps, endoscopic ultrasound (EUS)-guided fine-needle aspiration (FNA) and fine-needle core biopsy (FNB) can be performed for tissue sampling. EUS-FNA is widely used for tissue acquisition of SELs, however diagnostic yields for SELs vary and are relatively low from $74.5 \%$ to $83.9 \%$ [1 -4], particularly for small lesions that are technically challenging to sample using FNA. A prospective multicenter study by Eckardt et al revealed a low diagnostic yield of only $52 \%$ using a 19 G FNA needle [5]. While it had been hoped that the EUS Trucut biopsy needle (TCB) would confer superior results compared to EUS-FNA, there was no significant improvement for diagnosis of SELs in a large prospective study [6]. Recently, core biopsy using EUS-FNB has become available for sampling SELs, with diagnostic yields as high as $86 \%$ being achieved with high rates of histologic sampling [8-10]. However, a meta-analysis that examined the diagnos- tic yield and complication rates of EUS-FNA, TCB, and EUS-FNB for patients with gastrointestinal stromal tumors (GIST) demonstrated no significant differences between the techniques [10]. To improve diagnosis of SELs, several new tissue acquisition methods have been proposed, including suck-ligate-unroof-biopsy (SLUB) [11], use of endoscopic submucosal dissection (ESD) technique [12-14], endoscopic partial resection with unroofing technique (EPR-UT) [15], and single-incision needle-knife (SINK) biopsy [16]. When performing SINK, the overlying mucosa of a SEL is opened with a single linear incision using a standard needle knife and tissue samples are obtained by passing conventional biopsy forceps through the opening and deep into the lesion. In its initial description by de la Serna-Higuera et al, this technique provided tissue samples with a high diagnostic yield of $92 \%$, although only included 14 consecutive patients in their small series [16]. The aim of the current study was to further investigate the efficacy and safety of the SINK biopsy method in a larger patient sample. 


\section{Patients and methods}

We conducted a retrospective cohort study at a single tertiary referral center in Toronto, Canada. Patients referred to our institution with an upper gastrointestinal SEL from October 2013 to September 2015 were identified. Patients who underwent EUS followed by SINK biopsy were analyzed for diagnostic yield, including the rate of immunohistologic diagnosis, as well as for adverse events (AEs). Written and informed consent was obtained from each patient for all procedures. The study was carried out in accordance with the Declaration of Helsinki and was approved by the St. Michael's Hospital research ethics committee (REB\# 14-362).

\section{Procedures}

All procedures were performed with the patients under conscious sedation in the hospital endoscopy suite by 1 of 3 expert endoscopists (GM, JM, CT) ( Video). Esophagogastroduodenoscopy (EGD) (GIF-HQ190, GIF-1TH190; Olympus, Tokyo, Japan) was performed in all cases followed by examination with a radial (GF-UE160; Olympus) or linear echoendoscope (GFUCT140, GF-UCT180; Olympus). Pre-pathologic differential diagnoses based on lesion location, size, and layer of origin were recorded. Vascularity was assessed with pulsed Doppler. SELs larger than $10 \mathrm{~mm}$ with an intraluminal bulge that were deemed to require a tissue diagnosis to rule out a potentially malignant etiology such as GIST were selected for SINK biopsy at the endoscopist's discretion. Because this was a retrospective case series, there were no a priori exclusion criteria for study inclusion if the clinical decision to perform a SINK biopsy had been made. However, if there were obvious EUS features consistent with extrinsic compression, lipomas, cysts or varices, no tissue biopsies were performed. SELs without a prominent intraluminal bulge were sampled using EUS-FNA or FNB rather than SINK, and therefore, were not included in this analysis. While the pretest probability that esophageal SELs could be GISTs was unlikely, cases of SINK biopsy that were performed in the esophagus at the discretion of the endsocopist were included in the analysis because the primary purpose of this study was to examine the SINK biopsy technique itself rather than the underlying pathology.

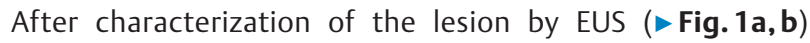
SINK biopsy was then performed with a forward viewing endoscope (GIF-1TH190; Olympus) or side viewing duodenoscope (TJF-Q180V; Olympus). Under direct endoscopic visualization, a $10 \mathrm{~mm}$ linear incision was made to the surface of the SEL using a conventional needle-knife sphincterotome with standard blended electrocautery (Endocut I: effect 2, duration 2, interval 3-ERBE VIO 300D, USA, Marietta, GA) (\$Fig.1c,d). A conventional biopsy forceps was introduced through the incision deep into the mass to obtain 3 to 6 samples. There was no onsite pathologist available for assessment of whether the specimens obtained were sufficient, therefore, the number of biopsies taken was determined at the clinical discretion of the endoscopist. The incision was closed using 1 to 2 endoclips for prophylaxis against subsequent bleeding. All patients were closely monitored in the endoscopy unit for 2 to 3 hours after the procedure. AEs were ascertained in the endoscopy unit prior to discharge and upon follow-up review. Biopsy samples were fixed in formalin and stained with hematoxylin-eosin for histological examination. When cell type, cellularity, cytoplasmic features, and nuclear atypia were compatible with mesenchymal origin, immunohistochemisty was performed to differentiate GIST from non-GIST lesions.

\section{Outcomes}

The primary outcome was diagnostic yield of the biopsy procedure, defined as the proportion of patients for whom the pathologist could provide a definitive diagnosis based on the specimen provided. A secondary outcome was the proportion of biopsy specimens for which immunohistochemistry could be successfully performed. Additional secondary outcomes included diagnostic rates in patients with small lesions $<2 \mathrm{~cm}$ or with previous non-diagnostic biopsies, as well as AEs. Perforation and major bleeding requiring blood transfusion, hospital admission, endoscopic reintervention or surgery were considered major complications. All other bleeding was considered a minor complication, which itself was classified as either moderate or minor. Minor bleeding was defined as intra-procedural oozing at the incision site that was easily managed by placement of clips. Moderate bleeding was defined as active bleeding refractory to clip placement that required additional hemostasis therapy during the index endoscopy procedure.

\section{Results}

\section{Patients}

A total of 49 patients underwent 50 SINK biopsies during the study period. The median age was 62 years (range: $33-88$ ); $61 \%(30 / 49)$ were male. All patients had prior EGD with $53 \%$ (26/49) having had at least 1 previous non-diagnostic tissue sampling ( 1 SINK biopsy, 10 EUS-FNA and 15 tunnelled biopsies). Location of the lesions were esophagus (5/49), gastroesophageal junction (9/49), stomach (32/49), and duodenum $(3 / 49)$.

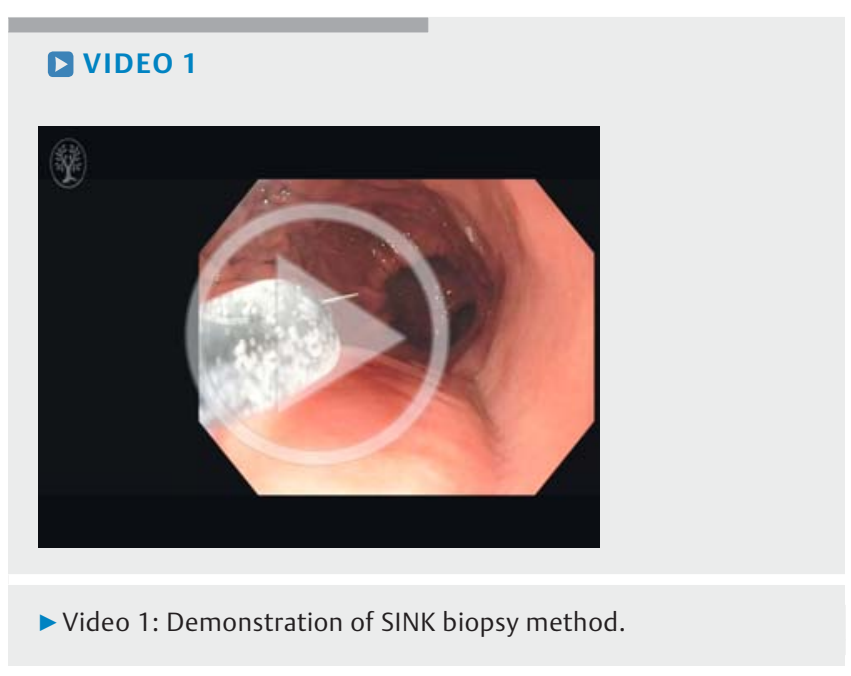



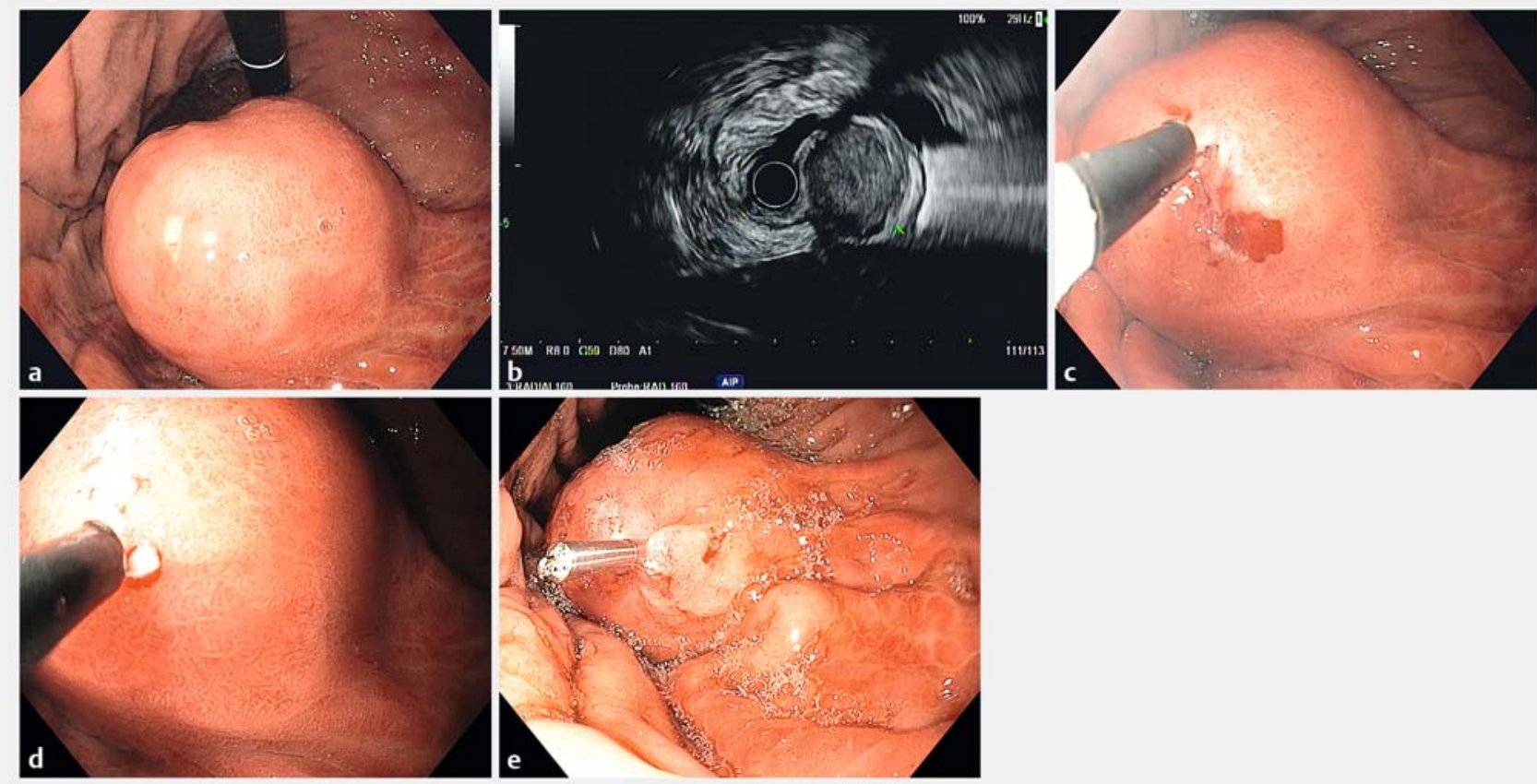

- Fig. 1 a Endoscopic evaluation of a subepithelial lesion. b Assessment by EUS. c, d A linear incision is made in the surface of the lesion using a conventional needle-knife sphincterotome. e Clip closure of the defect following biopsy sampling.

\section{Diagnostic yield}

Sufficient sampling for a definite pathologic diagnosis was obtained by SINK biopsy in 42 cases (86\%), with $91 \%(40 / 44)$ cases having sufficient sample to perform immunohistochemistry when deemed clinically relevant. Summaries of the lesion characteristics with specific final diagnoses are shown in $\bullet$ Table 1 and $>$ Table 2 . The pathologic diagnosis altered the pre-pathologic clinical diagnosis in 10 cases $(20 \%)$. There was no difference in diagnostic rates in small lesions $(<20 \mathrm{~mm})$ compared with larger lesions (>20 mm); $89 \%(16 / 18)$ and $87 \%(27 / 31)$ respectively $(P=0.63)$. Of the 26 patients with prior non-diagnostic biopsies or FNA, a specific diagnosis was obtained in $85 \%(22 / 26)$, including in $90 \%(9 / 10)$ of patients with past non-diagnostic FNA, $80 \%(12 / 15)$ of patients with past non-diagnostic tunnelled biopsy and 1 patient with past non-diagnostic SINK biopsy. Non-diagnostic cases with SINK biopsy were due to insufficient sampling of the targeted lesion in 7 cases. Of these 7 cases, 3 lesions were spindle cell lesions with inconclusive immunohistochemistry staining, 2 lesions were indefinite for leiomyoma due to scant smooth muscle present, and 2 lesions were suspected pancreatic rests that had insufficient abnormal tissue to definitively confirm the diagnosis.

\section{Complications}

In all cases, prophylactic closure of the incision site was attempted using endoclips. One incision could not be closed due to difficult mucosal apposition overlying a firm 2.6-cm SEL in the proximal stomach. Immediate bleeding at the site of the incision was common, which we considered minor bleeding, but in all cases was without clinical consequence. Four patients
(8\%) required endoscopic therapy using 1:20000 epinephrine injection and/or bipolar probe coagulation (Gold Probe; Boston Scientific, Natick, MA: 30 W ERBE bipolar coagulation). The remaining patients $(45 / 49)$ had no bleeding after application of endoclips. There were no major complications such as perforations or delayed procedure-related complications. All patients were discharged home following observation in the recovery room.

\section{Discussion}

Accurate diagnosis of SEL is often difficult as overlying mucosa may be normal and biopsy with conventional forceps is limited by its difficulty in accessing the underlying neoplasm for histopathology. To overcome these challenges, EUS-FNA is commonly performed to diagnose SEL. However, previous reports demonstrate diagnostic yields of only $60 \%$ to $80 \%$, with particular difficulty achieving a tissue diagnosis for small lesions [14]. The problem is that FNA obtains cellular aspirates that are typically inadequate for immunohistochemical staining, which is often required to reach a definitive diagnosis. Although there are recent studies reporting high diagnostic rates with FNB, a meta-analysis by Zhang et al comparing the diagnostic yield and complications of EUS-FNA, TCB, and FNB for patients with upper SEL demonstrated no significant differences between these methods [10].

In this study we report the largest series using the SINK biopsy method as an alternative tissue sampling technique, achieving a definite diagnostic rate of $86 \%$. Furthermore, a specific diagnosis was obtained in $85 \%$ of patients with prior non-diag- 
Table 1 Patient demographics and characteristics of subepithelia lesions.

\begin{tabular}{|c|c|}
\hline Age (yrs): Median (range) & $62(45-88)$ \\
\hline \multicolumn{2}{|l|}{$\operatorname{Sex}(\%)$} \\
\hline Male & $30(61.2)$ \\
\hline Female & $19(38.8)$ \\
\hline \multicolumn{2}{|l|}{ Tumor Location (\%) } \\
\hline Esophagus & $5(10.2)$ \\
\hline Stomach & $41(83.7)$ \\
\hline Cardia & $13(26.5)$ \\
\hline Fundus & $4(8.1)$ \\
\hline Body & $15(30.6)$ \\
\hline Antrum & $9(18.4)$ \\
\hline Duodenum & $3(6.1)$ \\
\hline Tumor size on EUS (mm): Median (range) & $24(10-80)$ \\
\hline \multicolumn{2}{|l|}{ Originating layer on EUS (\%) } \\
\hline $2^{\text {nd }}$ layer & $7(14.2)$ \\
\hline $2^{\text {nd }} / 3^{\text {rd }}$ layer & $4(8.2)$ \\
\hline $3^{\text {rd }}$ & $1(2.0)$ \\
\hline $3^{\text {rd }} / 4^{\text {th }}$ layer & $1(2.0)$ \\
\hline $4^{\text {th }}$ layer & $36(73.4)$ \\
\hline \multicolumn{2}{|l|}{ Prior sampling (\%) } \\
\hline No prior sampling & $21(42.9)$ \\
\hline Biopsies & $14(28.6)$ \\
\hline FNA & $12(24.5)$ \\
\hline Biopsies/FNA & $1(2.0)$ \\
\hline SINK & $1(2.0)$ \\
\hline Lesion < $20 \mathrm{~mm}$ & $18(36.7)$ \\
\hline
\end{tabular}

nostic biopsies or FNA. SINK is more likely to acquire adequate tissue for immunohistochemical staining, which may potentially overcome a limitation of EUS-FNA, TCB, and FNB. In addition, we achieved high diagnostic rates of $89 \%$ even with lesions smaller than $20 \mathrm{~mm}$. In order to further optimize the diagnostic yield, it is important to consider factors that help with risk stratification of SELs. Previous studies have demonstrated that larger, heterogeneous lesions with internal cystic spaces and irregular outer margins on EUS are high risk for harboring malignancy [17]. In addition, the lesion location is an important variable that predicts its behavior. SELs in the esophagus are considered low risk compared to lesions in the stomach or duodenum. Our results showed that all 5 cases of esophageal SELs were leiomyomas. Although risk factor stratification to determine the pretest probability of malignancy is useful, tissue

\begin{tabular}{|l|c|}
\hline Table 2 Results of SINK biopsies. & \\
\hline Diagnostic rates (\%) & $44(89.8)$ \\
\hline Diagnosis & 15 \\
\hline GIST & 16 \\
\hline Leiomyoma & 3 \\
\hline Pancreatic rest & 1 \\
\hline Glomus tumor & 2 \\
\hline IFP & 1 \\
\hline Lipoma & 1 \\
\hline Duplication cyst & 2 \\
\hline Schwannoma & $5(10.2)$ \\
\hline Non-diagnostic & \\
\hline Suggestive & $2(4.0)$ \\
\hline Leiomyoma & $16 / 18(88.9)$ \\
\hline Diagnostic rates of lesions <20 mm (\%) & \\
\hline GIST, gastrointestinal stromal tumor; IFP, inflammatory fibroid polyp \\
\hline
\end{tabular}

biopsy confirmation is often insisted upon by referring doctors and patients.

There were several cases of non-diagnostic SINK biopsies in our series despite a technically successful biopsy procedure ( $\triangleright$ Table 3). We speculate that this may have been due to insufficient depth of incision, resulting in failure to adequately expose the subepithelial tumor for the biopsy forceps to reach. Sufficient exposure of the tumor itself is the key element for obtaining adequate tissue by SINK biopsy. To overcome this limitation, a deep initial incision with the needle knife is necessary, a factor that limits this technique to SELs that exhibit intraluminal growth because a deep incision is likely prohibited for lesions without a bulge into the lumen.

We did not encounter any significant complications such as perforation, pain or unexpected bleeding. Although we experienced minor bleeding after making the needle-knife incision, in almost all cases this was not clinically significant and was easily managed by closure of the defect with endoclips following the biopsy sampling. No patients required hospital admission or had subsequent need for medical intervention due to bleeding.

Several other biopsy techniques for SELs have recently been proposed that may offer better access to the underlying tumor and potentially superior tissue acquisition. These new methods include suck-ligate-unroof-biopsy (SLUB) [11], block biopsy via submucosal endoscopy with a mucosal flap method (SEMF) [12 - 13], mucosal cutting biopsy [14], and endoscopic partial resection with the unroofing technique (EPR-UT) [15]. SLUB is a cap-based technique that involves suctioning the tissue into a cap and subsequently tightening a detachable loop to ligate the lesion. After ligation, EUS is applied to confirm loop placement below the SEL and the overlying mucosa is then unroofed using a needle knife. In contrast, SEMF utilizes ESD techniques to directly visualize the lesion through a dissected submucosal 
- Table 3 Characteristics of non-diagnostic lesions.

\begin{tabular}{|l|l|l|l|}
\hline Location & Size $(\mathbf{m m})$ & Wall layer (EUS) & Prior sampling \\
\hline Stomach (Antrum) & 80 & $4^{\text {th }}$ & FNA \\
\hline Stomach (body) & 30 & $4^{\text {th }}$ & Bx \\
\hline Stomach (Antrum) & 15 & $2^{\text {nd }}$ & Bx/FNA \\
\hline Stomach (body) & 15 & $3^{\text {rd }}$ & Nil \\
\hline Stomach (Antrum) & 30 & $4^{\text {th }}$ & Bx- ND \\
\hline Stomach (body) & 28 & $4^{\text {th }}$ & Nil \\
\hline Stomach (body) & 30 & $4^{\text {th }}$ \\
\hline FNA, fine needle aspiration; Bx, biopsy; ND, non-diagnostic; D, diagnostic; GIST, gastrointestinal stromal tumor; IFP, inflammatory fibroid polyp \\
\hline
\end{tabular}

tunnel. The mucosal cutting biopsy technique consists of injecting saline and indigo carmine dye between the surface of the lesion and the mucularis mucosa and a mucosal opening is made using a needle knife. This is repeated until the lesion is reached. EPR-UT is a method to unroof the overlying mucosa with endoscopic partial resection in several fragments in order to expose the tumor. Then the specimen is obtained by directly grasping the tumor using a snare. All of these novel methods were reported in non-comparative studies with small sample sizes. However, each method obtained specimens that were sufficient for a definitive diagnosis with a high degree of rigor, achieving diagnostic yields of $100 \%$ for SLUB, $100 \%$ for SEMF, $100 \%$ for mucosal cutting biopsies; and $94 \%$ for EPR-UT. While these techniques seem to be very promising, most are technically challenging and involve deeper penetration into the tumor using advanced endoscopic and microsurgical techniques. Thus, it would seem that they are likely more time consuming and technically challenging than SINK, although this hypothesis requires formal evaluation by prospective comparative studies.

A major strength of this study is that it involves a relatively large number of cases compared to the previously mentioned studies that described the other, new diagnostic techniques for SELs. Furthermore, our clinical experience using the SINK biopsy method illustrates a number of attractive attributes. First, we achieved a high diagnostic yield for a definite pathologic diagnosis using the SINK biopsy method. While our study design prevents us from making comparisons to other biopsy techniques, the overall results are nonetheless very encouraging and support the conclusion that SINK is an effective biopsy strategy. In addition, it is a technically simple method that does not require additional submucosal tunneling or other ESD-derived maneuvers that likely increase the complexity of the procedure. SINK does not require any dedicated endoscopic or microsurgical equipment other than a standard needle knife and the tissue is acquired using conventional biopsy forceps. Furthermore, we speculate that SINK may outperform EUS-FNA/ FNB for lesions at the gastric fundus and duodenum where the success rates of diagnostic sampling with FNA are reduced, particularly since SINK can be easily performed regardless of the anatomic location of the lesion. Last, SINK appears to be a very safe procedure with no major complications such as perforation or significant bleeding encountered in our experience. However, there are some obvious limitations to this study. First, no comparison was made to EUS-FNA or FNB, which is the current biopsy standard. This reflects the retrospective nature of our study design, limiting our conclusions to hypothesis generation only. Second, we were unable to assess the agreement between the histopathologic findings and immunostaining derived from SINK biopsy specimens with subsequent surgically resected specimens for variables such as mitotic index. Finally, SINK biopsy may be poorly suited for diagnosis of exophytic lesions that may be more appropriately sampled by EUS-FNA or by newer methods such as SLUB or SEMF.

\section{Conclusion}

In summary, SINK biopsy is a promising method for obtaining a tissue diagnosis for SELs. The strength of SINK is its ease and technical simplicity, and it has potential to be an alternative first-line tissue acquisition strategy rather than EUS-FNA/FNB. Whether it is truly superior to other methods in terms of both efficacy and safety will need to be determined by randomized, controlled trials.

\section{Competing interests}

None

\section{References}

[1] Suzuki T, Arai M, Matsumura T et al. Factors Associated with Inadequate Tissue Yield in EUS-FNA for Gastric SMT. ISRN Gastroenterol 2011; 2011: 619128

[2] Mekky MA, Yamao K, Sawaki A et al. Diagnostic utility of EUS-guided FNA in patients with gastric submucosal tumors. Gastrointest Endosc 2010; 71: 913-919

[3] Sepe PS, Moparty B, Pitman MB et al. EUS-guided FNA for the diagnosis of GI stromal cell tumors: sensitivity and cytologic yield. Gastrointest Endosc 2009; 70: $254-261$

[4] Hoda KM, Rodriguez SA, Faigel DO. EUS-guided sampling of suspected Gl stromal tumors. Gastrointest Endosc 2009; 69: 1218-1223 
[5] Eckardt A], Adler A, Gomes EM et al. Endosonographic large-bore biopsy of gastric subepithelial tumors: a prospective multicenter study. Eur J Gastroenterol Hepatol 2012; 24: 1135-1144

[6] Fernández-Esparrach G, Sendino O, Solé M et al. Endoscopic ultrasound-guided fine-needle aspiration and trucut biopsy in the diagnosis of gastric stromal tumors: a randomized crossover study. Endoscopy 2010; 42: 292-299

[7] Kim GH, Cho YK, Kim EY. Korean EUS Study Group. et al. Comparison of 22-gauge aspiration needle with 22-gauge biopsy needle in endoscopic ultrasonography-guided subepithelial tumor sampling. Scand J Gastroenterol. 2014; 49: 347-354

[8] Han JP, Lee TH, Hong S] et al. EUS-guided FNA and FNB after on-site cytologic evaluation in gastric subepithelial tumors. J Dig Dis 2016; 17: $582-587$

[9] Lee M, Min BH, Lee $\mathrm{H}$ et al. Feasibility and diagnostic yield of endoscopic ultrasonography-guided fine needle biopsy with a new core biopsy needle device in patients with gastric subepithelial tumors. Medicine (Baltimore) 2015; 94: e1622

[10] Zhang XC, Li QL, Yu YF et al. Diagnostic efficacy of endoscopic ultrasound-guided needle sampling for upper gastrointestinal subepithelial lesions: a meta-analysis. Surg Endosc 2016; 30: 2431 -2341
[11] Binmoeller KF, Shah JN, Bhat YM et al. Suck-ligate-unroof-biopsy by using a detachable $20-\mathrm{mm}$ loop for the diagnosis and therapy of small subepithelial tumors. Gastrointest Endosc 2014; 79: 750 - 755

[12] Kobara H, Mori H, Fujihara $S$ et al. Bloc biopsy by using submucosal endoscopy with a mucosal flap method for gastric subepithelial tumor tissue sampling. Gastrointest Endosc 2012; 77: 141-145

[13] Kobara H, Mori H, Fujihara S et al. Endoscopically visualized features of gastric submucosal tumors on submucosal endoscopy. Endoscopy 2014; 46: (Suppl. 01): UCTN E660-661

[14] Kataoka M, Kawai T, Yagi K et al. Mucosal cutting biopsy technique for histological diagnosis of suspected gastrointestinal stromal tumors of the stomach. Dig Endosc 2013; 25: 274-280

[15] Lee CK, Chung IK, Lee SH et al. Endoscopic partial resection with the unroofing technique for reliable tissue diagnosis of upper GI subepithelial tumors originating from the muscularis propria on EUS. Gastrointest Endosc 2010; 71: 188-194

[16] de la Serna-Higuera C, Pérez-Miranda M, Díez-Redondo P et al. EUSguided single-incision needle-knife biopsy: description and results of a new method for tissue sampling of subepithelial GI tumors. Gastrointest Endosc 2011; 74: 672-676

[17] Brand B, Oesterhelweg L, Binmoeller KF et al. Impact of endoscopic ultrasound for evaluation of submucosal lesions in gastrointestinal tract. Dig Liver Dis 2002; 34: 290-297 